index LGD $(\mathrm{HR}=3.0 ; 95 \%$ CI 1.3-6.7; $\mathrm{p}=0.009)$, and multifocal LGD $(\mathrm{HR}=2.8 ; 95 \%$ CI $1.3-6.1 ; \mathrm{p}=0.007)$. With this model we stratified the cohort into high and low risk groups, which corresponded to an overall positive predictive value (PPV) 31\%, and negative predictive value (NPV) 96\% by year 5 of follow-up $(\mathrm{p}<0.0001)$. To test this stratification criteria, the model was then applied to the external validation cohort and accurately predicted risk groups in this set with PPV 29\%, NPV 93\% by year 5 of follow-up $(\mathrm{p}<0.001)$. The final validated model was embedded in a web-based tool to calculate and illustrate patient-specific risk (figure 1).

Conclusions We have designed and externally validated $w w w$. UC-CaRE.uk, a personalised AN risk prediction webtool to facilitate shared decision-making over the management of LGD.

\section{IBD CARE IN 2020: RESULTS FROM THE FIRST IBD UK PATIENT AND SERVICE BENCHMARKING TOOL}

\begin{abstract}
${ }^{1,2} \mathrm{R}$ Kapasi, ${ }^{2} \mathrm{~J}$ Glatter ${ }^{*},{ }^{2} \mathrm{G}$ Winsor, ${ }^{2} \mathrm{AH}$ Parekh, ${ }^{2} \mathrm{~S}$ Bassil, ${ }^{2} \mathrm{~J}$ Clifford, ${ }^{2} \mathrm{~S}$ Berry, ${ }^{2} \mathrm{R}$ Ainley, ${ }^{3} \mathrm{C}$ Andrews, ${ }^{1} \mathrm{G}$ Bell, ${ }^{1} \mathrm{G}$ Bhatnagar, ${ }^{3} \mathrm{~J}$ Blackwell, ${ }^{1} \mathrm{~S}$ Bloom, ${ }^{1} \mathrm{C}$ Bramwell, ${ }^{1} \mathrm{M}$ Brookes, ${ }^{1}$ SR Brown, ${ }^{1} \mathrm{~N}$ Burch, ${ }^{1} \mathrm{~A}$ Burman, ${ }^{1} \mathrm{~K}$ Crook, ${ }^{1} \mathrm{~F}$ Cummings, ${ }^{3} \mathrm{~L}$ Dobson, ${ }^{1} \mathrm{~J}$ Epstein, ${ }^{1} \mathrm{O}$ Faiz, ${ }^{1} \mathrm{R}$ Feakins, ${ }^{1} \mathrm{M}$ Fletcher, ${ }^{1} \mathrm{~V}$ Garrick, ${ }^{3} \mathrm{~B}$ Hayee, ${ }^{1} \mathrm{~K}$ Keetarut, ${ }^{1} \mathrm{U}$ Meade, ${ }^{1} \mathrm{R}$ Muhammed, ${ }^{1} \mathrm{~A}$ Murdock, ${ }^{1} \mathrm{~N}$ Posford, ${ }^{1} \mathrm{G}$ Rowse, ${ }^{1} \mathrm{P}$ Sagar, ${ }^{3} \mathrm{~J}$ Segal, ${ }^{3} \mathrm{C}$ Selinger, ${ }^{1} \mathrm{~A}$ St Clair-Jones, ${ }^{1} \mathrm{SA}$ Taylor, 'S Weaver, 'L Younge, ${ }^{1} \mathrm{~K}$ Barrett, ${ }^{1}$ Ian Arnott, ${ }^{1} \mathrm{AB}$ Hawthorne, ${ }^{3} \mathrm{CA}$ Lamb. ${ }^{1} \mathrm{IBD}$ UK Board; ${ }^{2}$ Crohn's and Colitis UK; ${ }^{3}$ IBD UK Task and Finish Group
\end{abstract}

\subsection{6/gutjnl-2020-bsgcampus. 18}

Introduction Aligned to the 2019 IBD Standards, IBD UK developed a national Benchmarking Tool comprising a Service Self-Assessment for healthcare professionals and a Patient Survey. The aim was to qualify current local service performance to facilitate future quality improvement.

Methods The IBD Patient Survey ran from 8/7/19-22/11/19, and predominantly focussed on patient experience in the preceding 12 months. The Service Self-Assessment ran from 1/10/ 19-31/1/20. Estimates were encouraged where formal audit data was not available.

Results 10222 patients and 166 IBD (Inflammatory Bowel Disease) services (134 adult, 32 paediatric) completed the tools. Services cared for a median of 2000 adult (IQR 1400-3500) or 165 paediatric (IQR 100-280) patients. 38\% (12/32) of paediatric and $21 \%(28 / 134)$ adult services saw $>90 \%$ of patients with suspected IBD within 4 weeks of referral. 54\% $(1133 / 2104)$ of recently diagnosed patients felt what mattered to them was taken into account when making decisions about treatment and care. During a flare 77\% (127/166) of services reported being able to respond to $>90 \%$ of patients contacting the IBD service advice line by the end of the next working day. 61\% (6174/10071) of patients reported having contacted their IBD service advice line. When admitted to hospital 54\% (89/166) of services reported $>90 \%$ of patients were seen within 24 hours of admission by an IBD specialist on a gastroenterology ward. For elective surgery 69\% (114/ 166) of services reported waiting times of $<18$ weeks. $84 \%$ $(8486 / 10052)$ of patients had contact with an IBD nurse specialist. 42\% (4036/9677) of patients believed their care to be well-coordinated between their GP and gastroenterologist. $30 \%(3021 / 9943)$ of patients discussed wider life goals and priorities as part of planning their care. 79\% (7658/9691) of patients reported a lack of opportunities to feedback on their care. The proportion of adult multidisciplinary teams meeting the IBD Standards WTE (Whole Time Equivalent) staffing recommendations were gastroenterologists 31\%; colorectal surgeons 16\%; IBD nurse specialists 14\%; pharmacists 13\%; dietitians 7\%; and psychologists 2\%; and radiologists $79 \%$.

Conclusions The IBD UK Benchmarking Tool provides location-matched service performance and patient experience data. To support and measure impact of quality improvement, the Tool will be repeated longitudinally.

\section{EXPERIENCE OF FAMILY PLANNING OF FEMALE PATIENTS DIAGNOSED WITH INFLAMMATORY BOWEL DISEASE: A QUALITATIVE STUDY}

${ }^{1}$ Rebecca Homer, ${ }^{2}$ Helen Steed, ${ }^{3}$ Tiffany Wade, ${ }^{2,4}$ Matthew Brookes, ${ }^{5}$ Christian Selinger, ${ }^{1}$ Satvinder Purewal, ${ }^{6}$ Sarah Chapman, ${ }^{3}$ Wladyslawa Czuber-Dochan*. 'University of Wolverhampton, Wolverhampton, UK; ${ }^{2}$ The Royal Wolverhampton NHS Trust, Wolverhampton, UK; ${ }^{3}$ King's College London, London, UK; ${ }^{4}$ University of Birmingham, Birmingham, UK; ${ }^{5}$ St James University Hospital, Leeds, UK; ${ }^{6}$ University of Bath, Bath, UK

\subsection{6/gutjnl-2020-bsgcampus.19}

Background Inflammatory bowel disease (IBD) is a chronic illness affecting patients in their childbearing years. The physical effects of IBD on fertility and pregnancy in IBD in remission (e.g. disease is well controlled during conception and throughout pregnancy) are similar to the normal population. However, many women with IBD have high pregnancy-related anxieties and are more likely not to have children compared to women without IBD. The reasons cited for not having children include high levels of pregnancy-related anxieties have been insufficiently explored. The study aimed to explore the lived experience of family planning of women with inflammatory bowel disease (IBD) and their partners with or without IBD, during the reproductive stages of pre-conception, pregnancy and the postnatal period.

Methods Descriptive phenomenology was used to conduct face-to-face in-depth individual interviews. Purposive sampling was used to select participants with a maximum variation of different demographic and clinical factors, e.g. age, sex, UC/ CD diagnosis, disease duration, surgery, geographic location. The NVivo 12 software programme was used to manage the data and Colaizzi's framework was utilised in thematic data analysis.

Results 24 participants (21 women $11 \mathrm{CD} / 10 \mathrm{UC}$ and 3 partners) were recruited from out-patient clinics (22 participants) or through the Crohn's and Colitis UK website (2 participants). Women, average age 31 years of age (range 27-38), were at different family planning stages: pre-conception 6 women (3 actively planning family and 3 voluntarily childless); pregnant 8 women and 2 partners; and postpartum 7 women and one partner. Three women's partners, age 32-39, were recruited allowing for additional perspective of the experience being captured. In total 19 hours of interviews data were collected.

Six themes were identified: i) being diagnosed and controlling IBD symptoms; ii) relationship and family planning; iii) sources of information; iv) worries and concerns about pregnancy; v) post pregnancy care and problems; vi) ways of improving care. Women in pre-pregnancy stage and pregnant expressed a need for more information around these themes: (i) their medication and the impact of IBD on the baby; (ii) the genetic risk of passing the disease on. While pregnant and during post-partum stage, women identified a greater need for practical advice and support in relation to breastfeeding and looking after the baby. 